\title{
BMJ
}

\section{Low glomerular filtration rate and risk of stroke: meta- analysis}

\author{
Meng Lee, visiting scholar and instructor, ${ }^{1,3}$ Jeffrey L Saver, director and professor, ${ }^{1}$ Kuo-Hsuan Chang, \\ instructor, ${ }^{4}$ Hung-Wei Liao, director, ${ }^{5}$ Shen-Chih Chang, epidemiologist, ${ }^{6}$ Bruce Ovbiagele, associate \\ professor $^{1,2}$
}

\begin{abstract}
'Stroke Center, 710 Westwood
Plaza, University of California, Los Angeles, CA 90095, USA

${ }^{2}$ Department of Neurology, University of California, Los Angeles

${ }^{3}$ Department of Neurology, Chang Gung Memorial Hospital at Chiayi, Chang Gung University College of Medicine, Taiwan

${ }^{4}$ Department of Neurology, Chang Gung Memorial Hospital at Linkou, Chang Gung University College of Medicine

${ }^{5}$ Ching-Ten Clinic, Taiwan

${ }^{6}$ Department of Epidemiology,

School of Public Health, University of California

Correspondence to: B Ovbiagele
\end{abstract}

Ovibes@mednet.ucla.edu

Cite this as: BMJ 2010;341:C4249 doi:10.1136/bmj.c4249

\section{ABSTRACT}

Objective To qualitatively and quantitatively investigate the link between a low estimated glomerular filtration rate (eGFR) at baseline and risk of future stroke.

Design Systematic review and meta-analysis of prospective studies.

Data sources PubMed (1966-October 2009) and Embase (1947-October 2009).

Selection criteria Inclusion criteria were studies that prospectively collected data within cohort studies or clinical trials, estimated glomerular filtration rate at baseline using the modification of diet in renal disease or Cockcroft-Gault equations, assessed incident stroke, had a follow-up of at least one year, and reported quantitative estimates of multivariate adjusted relative risk and $95 \%$ confidence interval for stroke associated with an eGFR of $60-90 \mathrm{ml} / \mathrm{min} / 1.73 \mathrm{~m}^{2}$ or $<60 \mathrm{ml} / \mathrm{min} / 1.73 \mathrm{~m}^{2}$.

Data abstraction Two investigators independently abstracted data from eligible studies. Estimates were combined using a random effects model. Heterogeneity was assessed by $P$ value of $X^{2}$ statistics and $I^{2}$. Publication bias was assessed by visual examination of funnel plots.

Results 21 articles derived from 33 prospective studies: 14 articles assessed eGFR $<60 \mathrm{ml} / \mathrm{min} / 1.73 \mathrm{~m}^{2}$ and seven assessed eGRF at both $<60 \mathrm{ml} / \mathrm{min} / 1.73 \mathrm{~m}^{2}$ and 60 $90 \mathrm{ml} / \mathrm{min} / 1.73 \mathrm{~m}^{2}$ for a total of 284672 participants (follow-up 3.2-15 years) with 7863 stroke events. Incident stroke risk increased among participants with an eGFR $<60 \mathrm{ml} / \mathrm{min} / 1.73 \mathrm{~m}^{2}$ (relative risk 1.43, 95\% confidence interval 1.31 to 1.57 ; P 0.001 ) but not among those with an eGFR of $60-90 \mathrm{ml} / \mathrm{min} / 1.73 \mathrm{~m}^{2}$ (1.07, 0.98 to 1.17 ; $\mathrm{P}=0.15)$. Significant heterogeneity existed between estimates among patients with an eGFR $<60 \mathrm{ml} / \mathrm{min} / 1.73$ $\mathrm{m}^{2}$ (P<0.001). In subgroup analyses among participants with an eGFR $<60 \mathrm{ml} / \mathrm{min} / 1.73 \mathrm{~m}^{2}$, heterogeneity was significant in Asians compared with non-Asians (1.96, 1.73 to $2.23 v 1.25,1.16$ to $1.35 ; \mathrm{P}<0.001)$, and those with an eGFR of $40-60 \mathrm{ml} / \mathrm{min} / 1.73 \mathrm{~m}^{2} v<40 \mathrm{ml} / \mathrm{min} / 1.73 \mathrm{~m}^{2}$ (1.28, 1.04 to $1.56 v 1.77,1.32$ to 2.38 ; P<0.01).

Conclusions A baseline eGFR $<60 \mathrm{ml} / \mathrm{min} / 1.73 \mathrm{~m}^{2}$ was independently related to incident stroke across a variety of participants and study designs. Prompt and appropriate implementation of established strategies for reduction of vascular risk in people with know renal insufficiency may prevent future strokes.

\section{INTRODUCTION}

Chronic kidney disease and cardiovascular disease are major public health problems worldwide and often share the same pathophysiological mechanisms. Indeed, the prevalence of traditional cardiovascular risk factors can be high in those with impaired kidney function, ${ }^{2}$ and most patients with an estimated glomerular filtration rate (eGFR) lower than $60 \mathrm{ml} / \mathrm{min} / 1.73$ $\mathrm{m}^{2}$ die of cardiovascular causes and not progression to end stage renal disease. ${ }^{3}$ As such, averting future vascular events in patients with a low eGFR should be a primary goal. ${ }^{4}$

A systematic review of observational studies showed that a reduced eGFR was associated with an increased risk of coronary heart disease,${ }^{5}$ and a recent meta-analysis showed that a low eGFR was linked to all cause and cardiovascular mortality in the general population. ${ }^{6}$ The effect of reduced eGFR on incident stroke, however, has not been well delineated in a qualitative or quantitative manner using the totality of published data. As stroke is a leading cause of mortality and morbidity worldwide, and several strategies, such as blood pressure control and use of statins and aspirin, may reduce subsequent cardiovascular disease in patients with chronic kidney disease, it is important to identify people at potential high risk, then appropriate therapy can be applied. ${ }^{78}$ We carried out a systematic review and meta-analysis to determine whether a link exists between reduced eGFR and incident stroke and the magnitude of any relation.

\section{METHODS}

The search strategy was done according to the recommendations of the Meta-analysis of Observational Studies in Epidemiology. ${ }^{9}$ We searched PubMed (1966 to October 2009) and Embase (1947 to October 2009) using the search strategy "glomerular filtration rate" OR "renal disease" OR "chronic kidney disease" AND "stroke" OR "cerebrovascular disease" OR "cerebrovascular attack" OR "cerebral infarct" OR "intracranial hemorrhage" AND "prospective" OR 
"cohort" OR “observational" OR "post hoc" (see web extra fig 1). We restricted the search to studies in humans. No language restrictions were applied. Further information was retrieved through a manual search of references from recent reviews and relevant published original studies.

\section{Study selection and data abstraction}

We included studies that prospectively collected data within cohort studies or clinical trials, used the modification of diet in renal disease or Cockcroft-Gault equations to estimate glomerular filtration rate at baseline, assessed incident stroke, had a follow-up of at least one year, and reported quantitative estimates of the multivariate adjusted relative risk and $95 \%$ confidence interval for stroke associated with an eGFR of $60-90 \mathrm{ml} /$ $\min / 1.73 \mathrm{~m}^{2}$ or $<60 \mathrm{ml} / \mathrm{min} / 1.73 \mathrm{~m}^{2}$, or both. We excluded studies that had a cross sectional, case-control, or retrospective cohort study; that had mostly participants with end stage renal disease (by history of dialysis or an eGFR $<15 \mathrm{ml} / \mathrm{min} / 1.73 \mathrm{~m}^{2}$ ) or kidney transplant; that only reported unadjusted or age and sex adjusted relative risk; that did not report 95\% confidence intervals; and that were duplicated. Studies that used slightly varying eGFR intervals were included if they were otherwise comparable. Two investigators (ML and K-HC) independently abstracted data from eligible studies. Discrepancies were resolved by discussion with a third investigator $(\mathrm{BO})$ and by referencing the original report.

\section{Study quality}

We assessed the quality of eligible studies. Assessment was based on guidelines developed by the US Preventive Task Force as well as the modified checklist used in previous studies. ${ }^{10-12} \mathrm{We}$ assessed eight characteristics: prospective study design, maintenance of comparable groups, adjustment of potential confounders, documented loss of follow-up rate, assessor of outcome blinded to exposure status, clear definition of exposures (eGFR) and outcomes (stroke), temporality

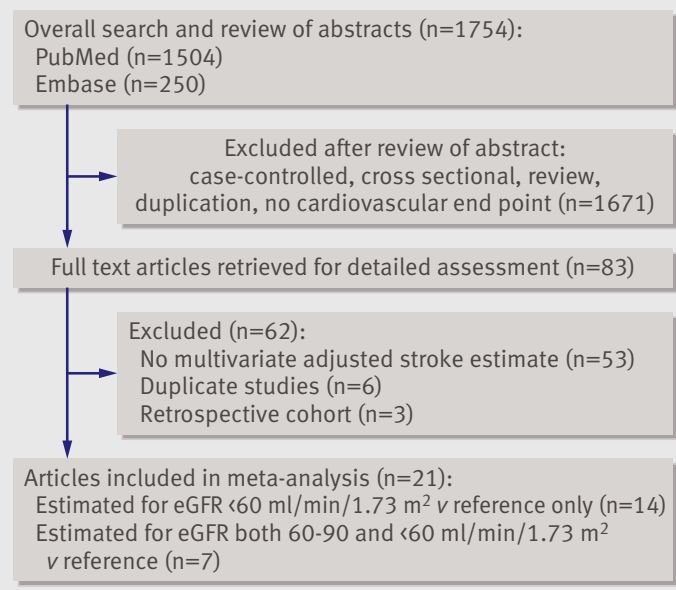

Fig 1| Flow of study selection
(eGFR measured at baseline, not at time of outcomes assessment), and follow-up of at least one year. Studies were graded as good quality if they met at least seven of eight criteria, fair if they met four to six, and poor if they met fewer than four.

\section{Statistical analysis}

For data analysis we used multivariate adjusted outcome data (expressed as relative risks and 95\% confidence intervals). When articles provided estimates based on both the modification of diet in renal disease and the Cockcroft-Gault equations, we used estimates from the more informative, expert recommended modification of diet in renal disease equation ${ }^{4}$ for primary analysis. In each study we converted these values by using their natural logarithms, and we calculated the standard errors from these logarithmic numbers and their corresponding 95\% confidence intervals. For the statistical analysis we combined log relative risks and standard errors using the inverse variance approach. We used a random effect model and explored for sources of inconsistency $\left(\mathrm{I}^{2}\right)$ and heterogeneity. A fixed effect model was also used for comparison with the random effects model on the overall risk estimate. Reported P values were two sided, with significance set at less than 0.05 . Heterogeneity was assessed by $\mathrm{P}$ value of $\chi^{2}$ statistics and $\mathrm{I}^{2}$, which describes the percentage of variability in the effect estimates that is due to heterogeneity rather than to chance. ${ }^{1314}$ Based on the suggestion of the Cochrane Collaboration we regarded heterogeneity as possibly unimportant when the $\mathrm{I}^{2}$ value was less than $40 \%$ and considerable when more than $75 \% .{ }^{15}$ RevMan 5 was used for the meta-analysis of observational studies. ${ }^{1617}$

The leading outcomes of interest were relative risks of incident stroke in patients with an eGFR of 60$90 \mathrm{ml} / \mathrm{min} / 1.73 \mathrm{~m}^{2}$ and $<60 \mathrm{ml} / \mathrm{min} / 1.73 \mathrm{~m}^{2}$. Publication bias was assessed by visual examination of funnel plots. Subgroup analyses for eGFRs $<60 \mathrm{ml} / \mathrm{min} / 1.73$ $\mathrm{m}^{2}$ were done according to normal references (studies using an eGFR $>60 \mathrm{ml} / \mathrm{min} / 1.73 \mathrm{~m}^{2}$ as the normal reference versus studies using $>90 \mathrm{ml} / \mathrm{min} / 1.73 \mathrm{~m}^{2}$ as normal), study population type (general or hypertension only versus established cardiovascular disease or high cardiovascular risk at entry), study design (ordinary cohorts versus secondary analysis of clinical trials), ethnicity (Asians $v$ non-Asians), follow-up $(<7$ years $v$ $\geq 7$ years), number of participants $(<10000 v \geq 10000)$, equation used to determine eGFR (modification of diet in renal disease $v$ Cockcroft-Gault), end points (fatal $v$ fatal plus non-fatal stroke), stroke subtype (ischaemic $v$ haemorrhagic stroke), sex (men $v$ women), degree of eGFR impairment (eGFR 40-60 $\mathrm{ml} / \mathrm{min} / 1.73 \mathrm{~m}^{2}$ or nearest equivalent $v<40 \mathrm{ml} / \mathrm{min} / 1.73 \mathrm{~m}^{2}$ or nearest equivalent), level of adjustment (age and sex adjusted $v$ multivariate adjusted), and study quality (good $v$ fair). We also explored the interaction between eGFR and albuminuria by using as a reference those groups with an $\mathrm{eGFR}$ of $>60 \mathrm{ml} / \mathrm{min} / 1.73 \mathrm{~m}^{2}$ without albuminuria. 


\section{Characteristics of included studies}

\begin{tabular}{|c|c|c|c|c|c|c|c|c|c|c|c|}
\hline $\begin{array}{l}\text { Study, } \\
\text { country }\end{array}$ & $\begin{array}{c}\text { Study } \\
\text { population }\end{array}$ & $\begin{array}{c}\text { Equation to } \\
\text { calculate } \\
\text { eGFR }\end{array}$ & $\begin{array}{c}\text { eGFR } \\
\text { groups (ml/ } \\
\min / 1.73 \\
\mathrm{~m} 2)\end{array}$ & $\begin{array}{l}\text { No of } \\
\text { partici- } \\
\text { pants }\end{array}$ & $\begin{array}{c}\% \\
\text { men }\end{array}$ & $\begin{array}{l}\text { Mean (SD) or } \\
\text { median } \\
\text { (range) age } \\
\text { (years) }\end{array}$ & $\begin{array}{c}\text { No of } \\
\text { strokes }\end{array}$ & $\begin{array}{c}\text { Follow- } \\
\text { up } \\
\text { (years) }\end{array}$ & End points & Adjusted variables & $\begin{array}{l}\text { Study } \\
\text { quality }\end{array}$ \\
\hline $\begin{array}{l}\text { Bax 2008, } \\
\text { Nether- } \\
\text { lands }^{18}\end{array}$ & $\begin{array}{l}\text { Atherosclero- } \\
\text { tic vascular } \\
\text { disease or } \\
\text { cardiovascular } \\
\text { risk factors at } \\
\text { entry }\end{array}$ & $\begin{array}{l}\text { Modification } \\
\text { of diet in } \\
\text { renal } \\
\text { disease }\end{array}$ & $\begin{array}{c}\text { >90 } \\
\text { (reference); } \\
60-90 ;<60\end{array}$ & $\begin{array}{c}\text { 602; } 2097 ; \\
517\end{array}$ & $\begin{array}{c}83 ; 77 ; \\
64\end{array}$ & $\begin{array}{l}54(10) ; 60 \\
(10) ; 67(8)\end{array}$ & $15 ; 59 ; 38$ & 3.3 & All stroke & $\begin{array}{l}\text { Age, sex, body mass index, } \\
\text { hypertension, coronary heart } \\
\text { disease, cerebral disease, } \\
\text { peripheral artery disease, } \\
\text { abdominal aortic aneurysm, } \\
\text { diabetes mellitus, smoking, and } \\
\text { use of angiotensin converting } \\
\text { enzyme inhibitors and } \\
\text { angiotensin II antagonists }\end{array}$ & Fair \\
\hline
\end{tabular}

\begin{tabular}{|c|c|c|c|c|c|c|c|c|c|}
\hline $\begin{array}{l}\text { Bos } 2007 \text {, } \\
\text { Nether- } \\
\text { lands }^{19}\end{array}$ & $\begin{array}{l}\text { General, no } \\
\text { stroke at entry }\end{array}$ & $\begin{array}{l}\text { Cockcroft- } \\
\text { Gault }\end{array}$ & $\begin{array}{c}\geq 60 \\
\text { (reference); } \\
<60\end{array}$ & $2652 ; 2285$ & 40 & 69 (62 to 77) & 586 & 10.2 & $\begin{array}{l}\text { All stroke } \\
\text { (ischaemic } \\
\text { and }\end{array}$ \\
\hline
\end{tabular}

Age, sex, and propensity score Good

stroke at entry Gault (reference);

and

(systolic blood pressure, diastolic

blood pressure, antihypertensive

haemorrha- drug use, left ventricular

gic recorded hypertrophy, diuretic use, pack

separately) years of smoking, diabetes

mellitus, cholesterol level, high

density lipoprotein level, carotid

intima media thickness, uric acid,

$C$ reactive protein, previous

myocardial infarction, previous

atrial fibrillation, waist to hip ratio,

antithrombotic drug use, lipid

lowering drug use)

\begin{tabular}{|c|c|c|c|c|c|c|c|c|c|c|c|}
\hline $\begin{array}{l}\text { Cheng 2008, } \\
\text { Taiwan }^{20}\end{array}$ & General & $\begin{array}{l}\text { Modification } \\
\text { of diet in } \\
\text { renal } \\
\text { disease }\end{array}$ & $\begin{array}{c}>90 \\
\text { (reference); } \\
60 \text { to } 90 ;<60\end{array}$ & $\begin{array}{c}4190 ; 11 \\
583 ; 1253\end{array}$ & $\begin{array}{c}63 ; 80 \\
87\end{array}$ & $\begin{array}{c}56(5) ; 57(5) \\
61(6)\end{array}$ & $29 ; 88 ; 35$ & 15 & $\begin{array}{l}\text { Fatal stroke } \\
\text { (ischaemic } \\
\text { and } \\
\text { haemorrha- } \\
\text { gic recorded } \\
\text { separately) }\end{array}$ & $\begin{array}{l}\text { Age, sex, body mass index, } \\
\text { smoking status (current, former, } \\
\text { never), total cholesterol level, } \\
\text { haemoglobin concentration, } \\
\text { diabetes mellitus, systolic blood } \\
\text { pressure, history of hypertension, } \\
\text { and prevalent cardiovascular } \\
\text { disease }\end{array}$ & Fair \\
\hline
\end{tabular}

\begin{tabular}{|c|c|c|c|c|c|c|c|c|c|}
\hline $\begin{array}{l}\text { Deo } 2008 \text {, } \\
\text { USA }^{21}\end{array}$ & $\begin{array}{l}\text { General, no } \\
\text { stroke at entry }\end{array}$ & $\begin{array}{l}\text { Modification } \\
\text { of diet in } \\
\text { renal } \\
\text { disease }\end{array}$ & $\begin{array}{c}\geq 60 \\
\text { (reference); } \\
<60\end{array}$ & $2340 ; 632$ & 49 & 74 (70 to 79$)$ & $126 ; 37$ & 6 & All stroke \\
\hline
\end{tabular}

Race, age, sex, site, body mass Fair

index, alcohol use, current

smoking status, diabetes

mellitus, hypertension, aspirin

use, diuretic use, angiotensin

converting enzyme inhibitors use,

$\beta$ blocker use, statin use, low

density lipoprotein and high

density lipoprotein cholesterol

level, plasminogen activator

inhibitor, $C$ reactive protein,

albumin, interleukin-6, and

tumour necrosis factor $\alpha$

\begin{tabular}{|c|c|c|c|c|c|c|c|c|c|c|c|}
\hline $\begin{array}{l}\text { Ford 2009, } \\
\text { Ireland, } \\
\text { Scotland, and } \\
\text { Nether- } \\
\text { lands }^{22}\end{array}$ & $\begin{array}{l}\text { Pre-existing } \\
\text { vascular } \\
\text { disease or } \\
\text { increased risk } \\
\text { of such } \\
\text { disease, } \\
\text { secondary } \\
\text { analysis of } \\
\text { clinical trial }\end{array}$ & $\begin{array}{l}\text { Modification } \\
\text { of diet in } \\
\text { renal } \\
\text { disease }\end{array}$ & $\begin{array}{c}\geq 60 \\
\text { (reference); } \\
50-60 ; 40- \\
50 ; 20-40\end{array}$ & $\begin{array}{c}2702 ; 1641 ; \\
1104 ; 349\end{array}$ & $\begin{array}{l}58 ; 48 ; \\
33 ; 26\end{array}$ & $\begin{array}{l}75(3) ; 75(3) ; \\
76(3) ; 77(3)\end{array}$ & $\begin{array}{c}190 ; 120 ; \\
74 ; 31\end{array}$ & 3.2 & $\begin{array}{l}\text { All stroke } \\
\text { and } \\
\text { transient } \\
\text { ischaemic } \\
\text { attacks }\end{array}$ & $\begin{array}{l}\text { Randomised treatment; country; } \\
\text { sex; current smoking status; age; } \\
\text { histories of hypertension, } \\
\text { diabetes mellitus, and vascular } \\
\text { disease; levels of low density } \\
\text { lipoprotein cholesterol and high } \\
\text { density lipoprotein cholesterol; } \\
\text { systolic and diastolic blood } \\
\text { pressure; glucose level; body } \\
\text { mass index; and C reactive protein }\end{array}$ & Good \\
\hline $\begin{array}{l}\text { Go } 2009, \\
\text { USA }^{23}\end{array}$ & $\begin{array}{l}\text { Atrial } \\
\text { fibrillation at } \\
\text { entry }\end{array}$ & $\begin{array}{l}\text { Modification } \\
\text { of diet in } \\
\text { renal } \\
\text { disease }\end{array}$ & $\begin{array}{c}\geq 60 \\
\text { (reference); } \\
45 \text { to } 59 ;<45\end{array}$ & $\begin{array}{c}7690 ; 2499 ; \\
1338\end{array}$ & $\begin{array}{c}60 ; 48 \\
52\end{array}$ & $\begin{array}{l}72 \text { (64 to } 78) ; \\
76 \text { (70 to } 82) ; \\
78 \text { (73 to } 83)\end{array}$ & 637 & 8 & $\begin{array}{l}\text { Thromboem- } \\
\text { bolic events, } \\
94 \% \text { were } \\
\text { ischaemic } \\
\text { stroke }\end{array}$ & $\begin{array}{l}\text { Age, sex, race/ethnicity, } \\
\text { educational attainment, annual } \\
\text { income status, previous } \\
\text { ischaemic stroke, heart failure, } \\
\text { diabetes mellitus, hypertension, } \\
\text { and coronary artery disease }\end{array}$ & Good \\
\hline $\begin{array}{l}\text { Irie } 2006 \text {, } \\
\text { Japan }{ }^{24}\end{array}$ & $\begin{array}{l}\text { General, men; } \\
\text { general, } \\
\text { women }\end{array}$ & $\begin{array}{l}\text { Modification } \\
\text { of diet in } \\
\text { renal } \\
\text { disease }\end{array}$ & $\begin{array}{l}\text { Men: } \geq 100 \\
\text { (reference), } \\
60 \text { to } 99,<60 \text {. } \\
\text { Women: } \\
\geq 100 \\
\text { (reference), } \\
60 \text { to } 99,<60\end{array}$ & $\begin{array}{c}\text { Men: } 7082 \text {, } \\
23858,824 . \\
\text { Women: } 10 \\
554,48041 \text {, } \\
2073\end{array}$ & $\begin{array}{l}\text { Men: } \\
100 \text { for } \\
\text { all } \\
\text { group- } \\
\text { S. } \\
\text { Wo- } \\
\text { men: } 0 \\
\text { for all } \\
\text { groups }\end{array}$ & 61 & $\begin{array}{c}\text { Men: } 84 \text {, } \\
\text { 363, } 44 . \\
\text { Women: } \\
53,365 \text {, } \\
76\end{array}$ & 10 & Fatal stroke & $\begin{array}{l}\text { Age, hypertension category, } \\
\text { cigarette smoking, alcohol intake, } \\
\text { diabetes mellitus, sex-specific } \\
\text { fifths of serum total cholesterol } \\
\text { level, serum high density } \\
\text { lipoprotein cholesterol level, body } \\
\text { mass index, and urinary protein }\end{array}$ & Fair \\
\hline $\begin{array}{l}\text { Kokubo 2009, } \\
\text { Japan } 25\end{array}$ & General & $\begin{array}{l}\text { Modification } \\
\text { of diet in }\end{array}$ & $\begin{array}{l}\quad \geq 90 \\
\text { (reference); }\end{array}$ & $\begin{array}{c}2415 ; 2452 \\
387 ; 124\end{array}$ & 47 & 56 & $\begin{array}{c}65 ; 99 ; 36 ; \\
13\end{array}$ & 11.7 & $\begin{array}{l}\text { All stroke } \\
\text { (ischaemic }\end{array}$ & $\begin{array}{l}\text { Age, sex, body mass index, } \\
\text { smoking, alcohol consumption, }\end{array}$ & Good \\
\hline
\end{tabular}




\begin{tabular}{|c|c|c|c|c|c|c|c|c|}
\hline $\begin{array}{l}\text { Study, } \\
\text { country }\end{array}$ & $\begin{array}{c}\text { Study } \\
\text { population }\end{array}$ & $\begin{array}{l}\text { Equation to } \\
\text { calculate } \\
\text { eGFR }\end{array}$ & $\begin{array}{c}\text { eGFR } \\
\text { groups (ml/ } \\
\min / 1.73 \\
\mathrm{~m} 2)\end{array}$ & $\begin{array}{l}\text { No of } \\
\text { partici- } \\
\text { pants }\end{array}$ & $\begin{array}{c}\% \\
\text { men }\end{array}$ & $\begin{array}{c}\text { Mean }(\mathrm{SD}) \text { or } \\
\text { median } \\
\text { (range) age } \\
\text { (years) }\end{array}$ & $\begin{array}{c}\text { No of } \\
\text { strokes }\end{array}$ & $\begin{array}{l}\text { Follow- } \\
\text { up } \\
\text { (years) }\end{array}$ \\
\hline & & $\begin{array}{l}\text { renal } \\
\text { disease }\end{array}$ & $\begin{array}{c}60 \text { to } 89 ; 50 \\
\text { to } 59 ;<50\end{array}$ & & & & & \\
\hline $\begin{array}{l}\text { Koren-Morag } \\
\text { 2006, Israel| }\end{array}$ & $\begin{array}{l}\text { Coronary heart } \\
\text { disease but } \\
\text { not stroke at } \\
\text { entry, } \\
\text { secondary } \\
\text { analysis of } \\
\text { clinical trial }\end{array}$ & $\begin{array}{l}\text { Modification } \\
\text { of diet in } \\
\text { renal } \\
\text { disease and } \\
\text { Cockcroft- } \\
\text { Gault }\end{array}$ & $\begin{array}{c}>60 \\
\text { (reference); } \\
\leq 60\end{array}$ & $5345 ; 1340$ & $91 ; 79$ & 58 (7); 65 (4) & $207 ; 80$ & 4.8 to 8.1 \\
\hline
\end{tabular}
and

haemorrhagic recorded separately)

schaemic

stroke and

transient

ischaemic

attacks
Adjusted variables

Study quality and present illness (hypertension, diabetes mellitus, and hypercholesterolaemia)

Age, sex, systolic blood pressure, Good

diabetes mellitus, level of triglycerides, high density lipoprotein level, New York Heart

Association functional class, body mass index, peripheral artery disease, current smoking status, antiplatelets, antihypertensive and lipid modifying drugs

Age, systolic blood pressure, Good antihypertensive treatment, smoking, body mass index, alcohol, exercise, total cholesterol level, $C$ reactive protein, use of hormone replacement therapy, diabetes mellitus, and assigned

entry,

secondary analysis of clinical trial

\begin{tabular}{|c|c|c|c|c|c|c|c|c|c|}
\hline $\begin{array}{l}\text { Nakayama } \\
\text { 2007, Japan }{ }^{28}\end{array}$ & General & $\begin{array}{l}\text { Cockcroft- } \\
\text { Gault }\end{array}$ & $\begin{array}{c}>70 \\
\text { (reference); } \\
40 \text { to } 70 ;<40\end{array}$ & $\begin{array}{c}555 ; 1246 \\
176\end{array}$ & $\begin{array}{c}42 ; 35 \\
35\end{array}$ & $\begin{array}{c}55(9) ; 65(7) \\
76(7)\end{array}$ & $15 ; 77 ; 20$ & 7.8 & All stroke \\
\hline
\end{tabular}

$\geq 90 \quad 14979 ; \quad 0$ forall $54(0.1) ; 55 \quad 197 ; 111 ; \quad 12 \quad$ All stroke $\begin{array}{rr}75 \text { to } 89 ; 60 & 1315\end{array}$ to $74 ;<60$ treatments

$57(0.2)$
Age, sex, systolic blood pressure, Good

body mass index, smoking status,

use of antihypertensive drugs,

\begin{tabular}{|c|c|c|c|c|c|c|c|c|c|}
\hline $\begin{array}{l}\text { Nickolas } \\
2008, \text { USA }^{29}\end{array}$ & $\begin{array}{l}\text { General, not } \\
\text { stroke at entry }\end{array}$ & $\begin{array}{l}\text { Cockcroft- } \\
\text { Gault }\end{array}$ & $\begin{array}{l}\quad \geq 60 \\
\text { (reference); } \\
15 \text { to } 59\end{array}$ & $2353 ; 945$ & 37 & 63 & 201 & 6.5 & All stroke \\
\hline
\end{tabular}
history of cardiovascular disease, hypercholesterolaemia, and diabetes mellitus

Age, sex, education,

hypertension, low density

lipoprotein cholesterol level, diabetes mellitus, prevalent cardiac disease, smoking, and alcohol consumption

\begin{tabular}{|c|c|c|c|c|c|c|c|c|c|c|c|}
\hline $\begin{array}{l}\text { Ninomiya } \\
\text { 2008,Japan } 30\end{array}$ & $\begin{array}{l}\text { General, data } \\
\text { from } 10 \\
\text { community- } \\
\text { based cohort } \\
\text { studies }\end{array}$ & $\begin{array}{l}\text { Modification } \\
\text { of diet in } \\
\text { renal } \\
\text { disease }\end{array}$ & $\begin{array}{l}\geq 90 \\
\text { (reference); } \\
60 \text { to } 89 ;<60\end{array}$ & $\begin{array}{c}7206 ; 14 \\
003 ; 1875\end{array}$ & $\begin{array}{c}39 ; 56 ; \\
5\end{array}$ & $58(12)$ & $\begin{array}{l}84 ; 404 ; \\
104\end{array}$ & 7.4 & All stroke & $\begin{array}{l}\text { Age, sex, cohort, systolic blood } \\
\text { pressure, diabetes mellitus, total } \\
\text { cholesterol level, body mass } \\
\text { index, and current smoking status }\end{array}$ & Fair \\
\hline $\begin{array}{l}\text { Perkovic } \\
2007, \\
\text { multicoun- } \\
\text { tries }^{38}\end{array}$ & $\begin{array}{l}\text { Stroke, } \\
\text { secondary } \\
\text { analysis of } \\
\text { clinical trial }\end{array}$ & $\begin{array}{l}\text { Cockcroft- } \\
\text { Gault }\end{array}$ & $\begin{array}{l}\geq 60 \\
\text { (reference); } \\
\quad<60\end{array}$ & $4314 ; 1757$ & $75 ; 55$ & $61(9) ; 70(8)$ & $460 ; 264$ & 4 & All stroke & $\begin{array}{l}\text { Age, sex, smoking status, } \\
\text { diabetes mellitus, systolic blood } \\
\text { pressure, body mass index, active } \\
\text { versus placebo therapy, and } \\
\text { single versus dual agent therapy }\end{array}$ & Good \\
\hline $\begin{array}{l}\text { Perticone } \\
2009 \text { Italy }^{31}\end{array}$ & $\begin{array}{l}\text { Postmenopau- } \\
\text { sal women, no } \\
\text { cardiovascular } \\
\text { disease or } \\
\text { diabetes } \\
\text { mellitus at } \\
\text { entry }\end{array}$ & $\begin{array}{l}\text { Modification } \\
\text { of diet in } \\
\text { renal } \\
\text { disease }\end{array}$ & $\begin{array}{l}\geq 60 \\
\text { (reference); } \\
\quad<60\end{array}$ & $1071 ; 429$ & $0 ; 0$ & $53(6) ; 53(6)$ & $41 ; 24$ & 6 & All stroke & $\begin{array}{l}\text { Age, smoking (former or never } \\
\text { smokers, current smokers), } \\
\text { cholesterol level, systolic blood } \\
\text { pressure, fasting glucose level, } \\
\text { body mass index, menopause, } \\
\text { and metabolic syndrome }\end{array}$ & Fair \\
\hline $\begin{array}{l}\text { Ruilope 2001, } \\
\text { Multicoun- } \\
\text { tries }^{32}\end{array}$ & $\begin{array}{l}\text { Hypertension } \\
\text { cohort, } \\
\text { secondary } \\
\text { analysis of } \\
\text { clinical trial }\end{array}$ & $\begin{array}{l}\text { Cockcroft- } \\
\text { Gault }\end{array}$ & $\begin{array}{l}>60 \\
\text { (eference); } \\
\quad \leq 60\end{array}$ & $\begin{array}{c}15770 \\
2821\end{array}$ & $57 ; 30$ & $60(7) ; 68(7)$ & $211 ; 77$ & 3.8 & All stroke & $\begin{array}{l}\text { Achieved diastolic and systolic } \\
\text { blood pressure, age, gender, } \\
\text { smoking habits, previous } \\
\text { cardiovascular disease, diabetes } \\
\text { mellitus, and total cholesterol }\end{array}$ & Good \\
\hline $\begin{array}{l}\text { Ruilope } 2007 \text {, } \\
\text { multicoun- } \\
\text { tries }^{33}\end{array}$ & $\begin{array}{l}\text { Hypertension } \\
\text { cohort, } \\
\text { secondary } \\
\text { analysis of } \\
\text { clinical trial }\end{array}$ & $\begin{array}{l}\text { Modification } \\
\text { of diet in } \\
\text { renal } \\
\text { disease and } \\
\text { Cockcroft- } \\
\text { Gault }\end{array}$ & $\begin{array}{l}\geq 60 \\
\text { (reference); } \\
\quad<60\end{array}$ & 9214; 5999 & $67 ; 44$ & 65 (8); 70 (8) & 603 & 4.6 & All stroke & $\begin{array}{l}\text { Age, sex, coronary heart disease, } \\
\text { and left ventricular hypertrophy }\end{array}$ & Good \\
\hline $\begin{array}{l}\text { Shilipak } \\
\text { 2001, USA }\end{array}$ & $\begin{array}{l}\text { Postmenopau- } \\
\text { sal women } \\
\text { with coronary } \\
\text { heart disease, } \\
\text { secondary } \\
\text { analysis of } \\
\text { clinical trial }\end{array}$ & $\begin{array}{l}\text { Cockcroft- } \\
\text { Gault }\end{array}$ & $\begin{array}{l}>60 \\
\text { (reference); } \\
40 \text { to } 60 ;<40\end{array}$ & $\begin{array}{l}1306 ; 1135 ; \\
322\end{array}$ & $\begin{array}{l}0 \text { for all } \\
\text { groups }\end{array}$ & $66(7)$ & $70 ; 93 ; 51$ & 4.1 & $\begin{array}{l}\text { All stroke } \\
\text { and } \\
\text { transient } \\
\text { ischaemic } \\
\text { attacks }\end{array}$ & $\begin{array}{l}\text { Age; race; hypertension; diabetes } \\
\text { mellitus; tobacco use; previous } \\
\text { coronary artery bypass surgery; } \\
\text { body mass index; waist to hip } \\
\text { ratio; levels of low density } \\
\text { lipoprotein cholesterol, high } \\
\text { density lipoprotein cholesterol, } \\
\text { triglyceride, and lipoprotein(a); } \\
\text { physical activity; lipid lowering }\end{array}$ & Good \\
\hline
\end{tabular}




\begin{tabular}{|c|c|c|c|c|c|c|c|c|c|c|c|}
\hline $\begin{array}{l}\text { Study, } \\
\text { country }\end{array}$ & $\begin{array}{l}\text { Study } \\
\text { population }\end{array}$ & $\begin{array}{c}\text { Equation to } \\
\text { calculate } \\
\text { eGFR }\end{array}$ & $\begin{array}{c}\text { eGFR } \\
\text { groups (ml/ } \\
\min / 1.73 \\
\mathrm{~m} 2)\end{array}$ & $\begin{array}{l}\text { No of } \\
\text { partici- } \\
\text { pants }\end{array}$ & $\begin{array}{c}\text { \% } \\
\text { men }\end{array}$ & $\begin{array}{l}\text { Mean (SD) or } \\
\text { median } \\
\text { (range) age } \\
\text { (years) }\end{array}$ & $\begin{array}{l}\text { No of } \\
\text { strokes }\end{array}$ & $\begin{array}{l}\text { Follow- } \\
\text { up } \\
\text { (years) }\end{array}$ & End points & Adjusted variables & $\begin{array}{l}\text { Study } \\
\text { quality }\end{array}$ \\
\hline & & & & & & & & & & $\begin{array}{l}\text { drug use; diuretic use; and atrial } \\
\text { fibrillation }\end{array}$ & \\
\hline $\begin{array}{l}\text { Tonelli 2006, } \\
\text { USA and } \\
\text { Canada }^{35}\end{array}$ & $\begin{array}{l}\text { History of } \\
\text { myocardial } \\
\text { infarction, } \\
\text { secondary } \\
\text { analysis of } \\
\text { clinical trial }\end{array}$ & $\begin{array}{l}\text { Modification } \\
\text { of diet in } \\
\text { renal } \\
\text { disease }\end{array}$ & $\begin{array}{c}\geq 60 \\
\text { (reference); } \\
<60\end{array}$ & $2839 ; 707$ & $89 ; 75$ & $\begin{array}{l}58 \text { (50 to } 64) ; \\
65 \text { (59 to } 70)\end{array}$ & $71 ; 28$ & 5 & All stroke & $\begin{array}{l}\text { Age, sex, ethnic origin, smoking } \\
\text { status, diabetes mellitus, waist to } \\
\text { hip circumference ratio, fasting } \\
\text { glucose level, haemoglobin } \\
\text { concentration, albumin, low } \\
\text { density lipoprotein and high } \\
\text { density lipoprotein cholesterol } \\
\text { levels, triglyceride levels, systolic } \\
\text { and diastolic blood pressure, } \\
\text { country of treatment (US } v \\
\text { Canada), left ventricular ejection } \\
\text { fraction, and use of drugs ( } \beta \\
\text { blockers, angiotensin converting } \\
\text { enzyme inhibitors, aspirin, or } \\
\text { pravastatin) }\end{array}$ & Good \\
\hline $\begin{array}{l}\text { Weiner 2004, } \\
\text { USA }^{36}\end{array}$ & $\begin{array}{l}\text { Combined four } \\
\text { population } \\
\text { studies } \\
\text { (Atherosclero- } \\
\text { sis Risk in } \\
\text { Community } \\
\text { Study, } \\
\text { Cardiovascular } \\
\text { Health Study, } \\
\text { Framingham } \\
\text { Heart Study, } \\
\text { and } \\
\text { Framingham } \\
\text { Offspring } \\
\text { Study) }\end{array}$ & $\begin{array}{l}\text { Modification } \\
\text { of diet in } \\
\text { renal } \\
\text { disease }\end{array}$ & $\begin{array}{l}\geq 60 \\
\text { (reference); } \\
15 \text { to } 59\end{array}$ & $\begin{array}{c}20970 ; \\
1664\end{array}$ & $44 ; 33$ & $\begin{array}{c}56(11) ; 68 \\
\text { (11) }\end{array}$ & $587 ; 125$ & 10 & All stroke & $\begin{array}{l}\text { Age, sex, hypertension, diabetes } \\
\text { mellitus, systolic blood pressure, } \\
\text { body mass index, total and high } \\
\text { density lipoprotein cholesterol } \\
\text { level, current smoking status, } \\
\text { current alcohol use, left } \\
\text { ventricular hypertrophy, high } \\
\text { school graduation status, and } \\
\text { race }\end{array}$ & Fair \\
\hline $\begin{array}{l}\text { Yang 2008, } \\
\text { China }^{37}\end{array}$ & $\begin{array}{l}\text { Diabetic } \\
\text { population } \\
\text { without stroke } \\
\text { at entry }\end{array}$ & $\begin{array}{l}\text { Modification } \\
\text { of diet in } \\
\text { renal } \\
\text { disease }\end{array}$ & $\begin{array}{l}\geq 115 \\
\text { (reference); } \\
60 \text { to } 114.9 ; \\
<60\end{array}$ & 6969 & 46 & 57 & 314 & 5.4 & $\begin{array}{l}\text { Ischaemic } \\
\text { stroke }\end{array}$ & $\begin{array}{l}\text { Age; sex; systolic and diastolic } \\
\text { blood pressure; haemoglobin } \mathrm{A}_{1 \mathrm{c}} \text {; } \\
\text { body mass index; haemoglobin } \\
\text { concentration; white blood cell } \\
\text { count; levels of high density } \\
\text { lipoprotein, low density } \\
\text { lipoprotein, total cholesterol, and } \\
\text { triglyceride; and drug use (blood } \\
\text { pressure lowering, cholesterol } \\
\text { lowering, insulin, antiplatelet, } \\
\text { angiotensin converting enzyme } \\
\text { inhibitor, and angiotensin II } \\
\text { antagonist) }\end{array}$ & Fair \\
\hline
\end{tabular}

\section{RESULTS}

The literature review identified 83 full articles for detailed assessment, of which 53 were excluded for having no multivariate adjusted stroke estimate, six for being duplicated studies, and three for having a retrospective cohort design. Our final primary analysis included 21 articles derived from 33 prospective studies $^{18-38}$ : 14 articles assessed eGFR $<60 \mathrm{ml} / \mathrm{min} /$ $1.73 \mathrm{~m}^{2}$ only and seven assessed both $<60 \mathrm{ml} / \mathrm{min} /$ $1.73 \mathrm{~m}^{2}$ and $60-90 \mathrm{ml} / \mathrm{min} / 1.73 \mathrm{~m}^{2}$ (fig 1 ). The table shows the characteristics of the included studies. Overall, 284672 participants had a total of 7863 stroke events. Among the 21 articles, one contained 10 community cohorts from Japan ${ }^{30}$ and the other four community cohorts from the United States. ${ }^{36}$ Participants were derived from ordinary cohorts in 13 articles and clinical trials in eight. On a scale of 8 the overall quality of studies was good (median score 7 , range 5-8).
Follow-up ranged from $3.2^{22}$ to 15 years. ${ }^{20}$ Glomerular filtration rate was estimated by the modification of diet in renal disease equation in 15 articles and by the Cockcroft-Gault equation in six. Nineteen articles reported fatal plus non-fatal stroke as a primary end point, whereas two reported fatal stroke as a primary end point. ${ }^{2024}$ One study used thromboembolic events as a primary end point, but ischaemic stroke constituted over $94 \%$ of total thromboembolic events. ${ }^{23}$ Transient ischaemic attacks were only included as end points in three studies. ${ }^{22634}$

\section{Main outcome}

Pooling results from the random effects model showed that incident stroke increased among patients with an eGFR $<60 \mathrm{ml} / \mathrm{min} / 1.73 \mathrm{~m}^{2}$ (relative risk 1.43, 95\% confidence interval 1.31 to $1.57, \mathrm{P}<0.001$; fig 2$)$. The risk of incident stroke did not, however, increase 
significantly among patients with an eGFR of 60$90 \mathrm{ml} / \mathrm{min} / 1.73 \mathrm{~m}^{2}$ (1.07, 0.98 to $1.17, \mathrm{P}=0.15$; fig 2$)$.

Significant heterogeneity existed between estimates

\section{Study}

eGFR $<60 \mathrm{ml} / \mathrm{min} / 1.73 \mathrm{~m}^{2} v$ reference

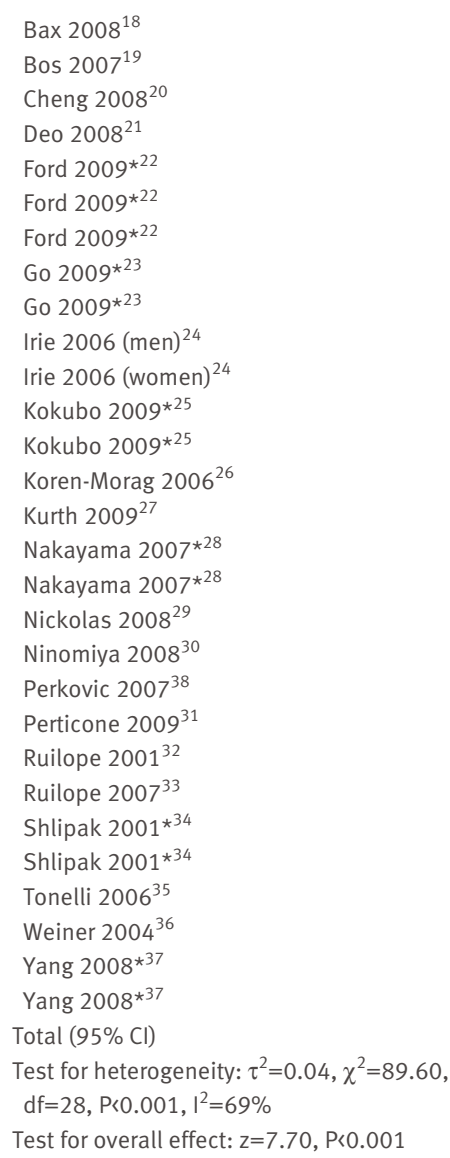

eGFR $60-90 \mathrm{ml} / \mathrm{min} / 1.73 \mathrm{~m}^{2} \mathrm{v}$ reference

\section{Bax $2008^{18}$}

Cheng $2008^{20}$

Irie 2006 (men) $\dagger^{24}$ Irie 2006 (men) $\dagger^{24}$ Irie 2006 (men) $\dagger^{24}$ Irie 2006 (men) $\mathrm{t}^{24}$ Irie 2006 (women) $\mathrm{t}^{24}$ Irie 2006 (women) $\mathrm{t}^{24}$ Irie 2006 (women) $\mathrm{t}^{24}$ Irie 2006 (women) $\mathrm{t}^{24}$ Kokubo $2009^{25}$ Kurth $2009 t^{27}$ Kurth $2009 t^{27}$ Ninomiya $2008^{30}$ Yang $2008 t^{37}$ Yang $2008 t^{37}$ Total $(95 \% \mathrm{Cl})$

Test for heterogeneity: $\tau^{2}=0.01, \chi^{2}=24.05$, $\mathrm{df}=15, \mathrm{P}=0.06, \mathrm{I}^{2}=38 \%$

Test for overall effect: $z=1.43, P=0.15$

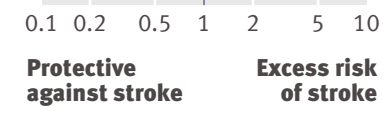

Fig 2 Risk ratio for association of estimated glomerular filtration rate (eGFR) and risk of stroke in prospective cohort studies. *Subgroups of estimates with eGFR $<60 \mathrm{ml} / \mathrm{min} / 1.73 \mathrm{~m}^{2}$.

†Subgroups of estimates with eGFR $60-90 \mathrm{ml} / \mathrm{min} / 1.73 \mathrm{~m}^{2}$ among patients with an eGFR $<60 \mathrm{ml} / \mathrm{min} / 1.73 \mathrm{~m}^{2}$ $\left(\mathrm{P}<0.001, \mathrm{I}^{2}=69 \%\right)$ but not among those with an eGFR of $60-90 \mathrm{ml} / \mathrm{min} / 1.73 \mathrm{~m}^{2}\left(\mathrm{P}=0.06, \mathrm{I}^{2}=38 \%\right)$. The estimates were similar between the fixed effects model and random effect model.

\section{Subgroup analyses}

An eGFR $<60 \mathrm{ml} / \mathrm{min} / 1.73 \mathrm{~m}^{2}$ was associated with an increased risk of subsequent stroke in all subgroups when estimates were stratified by eGFR reference group, study population type, study design, ethnicity, duration of follow-up, number of participants, equation used to determine eGFR, end points, sex, stroke subtype, different eGFR intervals $<60 \mathrm{ml} / \mathrm{min} / 1.73$ $\mathrm{m}^{2}$, study quality, and level of adjustment (fig 3). Significant heterogeneity between pooled analyses were noted for studies using an eGFR $>60 \mathrm{ml} / \mathrm{min} / 1.73 \mathrm{~m}^{2}$ as the normal reference compared with studies using $>90 \mathrm{ml} / \mathrm{min} / 1.73 \mathrm{~m}^{2}$ as normal $(1.29,1.18$ to $1.41 \mathrm{v}$ $1.82,1.53$ to $2.16, \mathrm{P}$ for heterogeneity among subgroups $<0.001)$, cohort studies compared with clinical trials $(1.59,1.40$ to $1.81 v 1.25,1.13$ to $1.38, \mathrm{P}<0.01)$, Asians compared with non-Asians (1.96, 1.73 to $2.23 v 1.26$, 1.16 to $1.35, \mathrm{P}<0.001)$, fatal compared with fatal plus non-fatal stroke $(1.97,1.63$ to $2.38 v 1.38,1.26$ to 1.51 , $\mathrm{P}<0.001)$, eGFR $40-60 \mathrm{ml} / \mathrm{min} / 1.73 \mathrm{~m}^{2} v<40 \mathrm{ml} / \mathrm{min} /$ $1.73 \mathrm{~m}^{2}(1.28,1.04$ to $1.56 v 1.77,1.32$ to $2.38, \mathrm{P}<0.01)$, and good study quality compared with fair study quality $(1.35,1.23$ to 1.49 v $1.62,1.33$ to $1.97, \mathrm{P}=0.01)$.

A total of 11 studies reported adjusted estimates of the strength of the association, first by age and sex then by other known cardiovascular risk factors - for example, blood pressure, smoking, lipids levels, diabetes. The overall age and sex adjusted summary estimate was $1.64(95 \%$ confidence interval 1.45 to 1.85$)$, which after further adjustment of other known cardiovascular risk factors was reduced to 1.45 (1.26 to 1.68; $\mathrm{P}$ for heterogeneity among subgroups 0.01 ).

Otherwise no obvious heterogeneity found between baseline risk populations (general or hypertension only $v$ high cardiovascular risk), duration of followup, number of participants, equation used to determine eGFR, stroke subtypes, and sex. Based on the few papers that provided information on the interaction between proteinuria and eGFR, proteinuria did not substantially increase the risk of stroke in patients with an eGFR of $<60$ or $>60 \mathrm{ml} / \mathrm{min} / 1.73 \mathrm{~m}^{2}$ (fig 4).

\section{Publication bias}

The funnel plots showed no major asymmetry except for a small degree of publication bias, with a slight under-representation of small studies showing neutral or unexpected protective effects (see web extra fig 2).

\section{DISCUSSION}

In this meta-analysis of 21 articles derived from 33 prospective studies of generally good quality, among over 280000 people experiencing almost 8000 stroke events, we found that patients with a baseline estimated glomerular filtration rate (eGFR) of $<60 \mathrm{ml} / \mathrm{min} / 1.73$ $\mathrm{m}^{2}$ had a risk of future stroke that was $43 \%$ greater than 


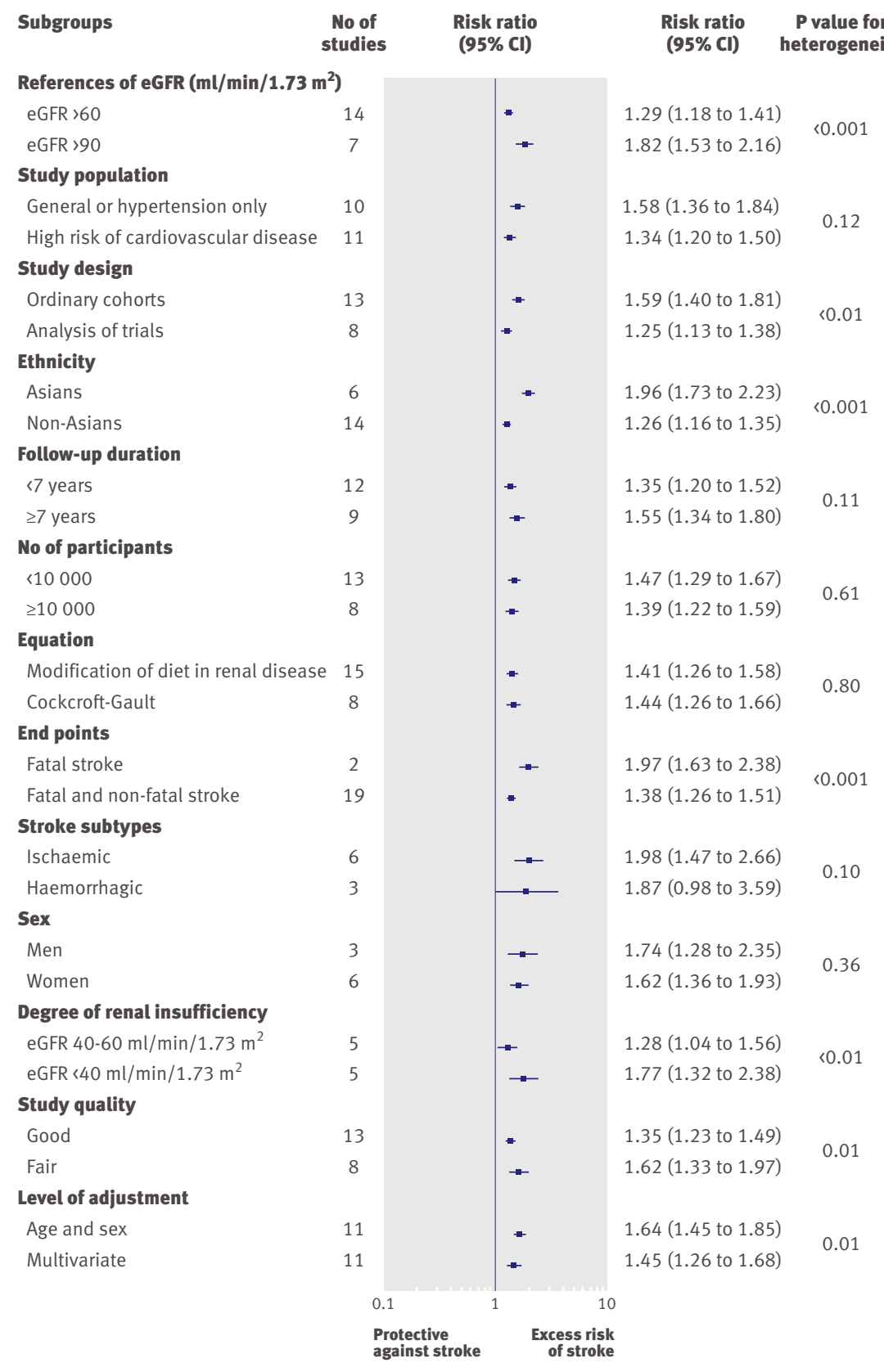

Fig 3 Subgroup analyses for comparison between studies reporting associations of estimated glomerular filtration rate (eGFR) $<60 \mathrm{ml} / \mathrm{min} / 1.73 \mathrm{~m}^{2}$ with risk of stroke

those with a normal baseline eGFR. This relation was consistent across diverse population subgroups-that is, those with or without traditional cardiovascular risk factors. The size and inclusion of only prospectively collected data strengthened the robustness of our findings, as selection bias, recall bias, and reverse causality were unlikely. In addition, all studies included in our meta-analysis reported a multivariate adjusted relative risk, which probably mitigated the possibility of known confounding influencing our results.

We used subgroup analyses to assess the varying influence of several factors on the association between eGFR $<60 \mathrm{ml} / \mathrm{min} / 1.73 \mathrm{~m}^{2}$ and risk of stroke. The magnitude of risk was larger when studies used an eGFR $>90 \mathrm{ml} / \mathrm{min} / 1.73 \mathrm{~m}^{2}$ as reference compared with $>60 \mathrm{ml} / \mathrm{min} / 1.73 \mathrm{~m}^{2}$, which raised the possibility that an eGFR of $60-90 \mathrm{ml} / \mathrm{min} / 1.73 \mathrm{~m}^{2}$ may increase the risk of stroke compared with an eGFR $>90 \mathrm{ml} /$ $\min / 1.73 \mathrm{~m}^{2}$. Our formal meta-analysis did not, however, show significantly increased risk of incident stroke among patients with an eGFR of $60-90 \mathrm{ml} / \mathrm{min} / 1.73 \mathrm{~m}^{2}$. The explanation could be that such a rate is not sensitive enough as a marker of kidney disease to discriminate risk of stroke. We did, however, find a possible dose-response relation between eGFR and stroke at levels $<60 \mathrm{ml} / \mathrm{min} /$ $1.73 \mathrm{~m}^{2}$ - that is, the risk of stroke was significantly greater for eGFR $<40 \mathrm{ml} / \mathrm{min} / 1.73 \mathrm{~m}^{2}$ than for levels of $40-60 \mathrm{ml} / \mathrm{min} / 1.73 \mathrm{~m}^{2}$.

A meta-analysis based on observational studies cannot prove causality. However, based on these results it may not be unreasonable to regard the presence of a low eGFR as a marker for increased risk of stroke, prompting optimal application of established vascular risk reduction strategies such as control of blood pressure, statin use, and antiplatelet therapy. ${ }^{7}$

Interestingly we found that Asian people with a low baseline eGFR seemed to be at higher risk of future stroke. Indeed, in Asian populations, hypertension is a major risk factor of both stroke and death from renal causes, ${ }^{3940}$ chronic kidney disease further increases the association of blood pressure with stroke, ${ }^{25}$ and metaanalysis showed that the risk of stroke associated with hypertension is consistently and significantly greater in Chinese than in white people. ${ }^{41}$ Furthermore, it has been suggested that Asian people tend to develop hypertension at earlier ages than other races ${ }^{42}$ and it is conceivable that a longer history of hypertension may cause more profound damage of end organs and vessels thereby leading to a higher likelihood of vascular events within a given study period. A systematic review that linked reduced eGFR with increased risk of coronary heart disease was only among participants in Western countries and so did not have the means of exploring this issue. ${ }^{5}$ Although most of the studies we analysed adjusted for hypertension or blood pressure, none adjusted for the duration of hypertension, thereby limiting the extent to which we could fully adjust for hypertension as a confounder. As such, this potential disparity between races will need to be more comprehensively explored in future studies.

We also observed that the effect of reduced eGFR was more profound on the risk of fatal stroke than on all strokes, which probably points to the association of compromised kidney function with risk factors for generally poor clinical outcomes such as oxidative stress, widespread inflammation, electrolyte derangements, procoagulation, and presence of uraemic toxins. ${ }^{3}$ In fact, kidney disease even of mild severity has been shown to be an independent predictor of poorer clinical outcomes among people with stroke, including higher risk of all cause mortality and cardiovascular mortality. ${ }^{4344}$ Also of note, the presence of albuminuria did not substantially further increase the risk of stroke among patients with a baseline eGFR of $<60$ or 


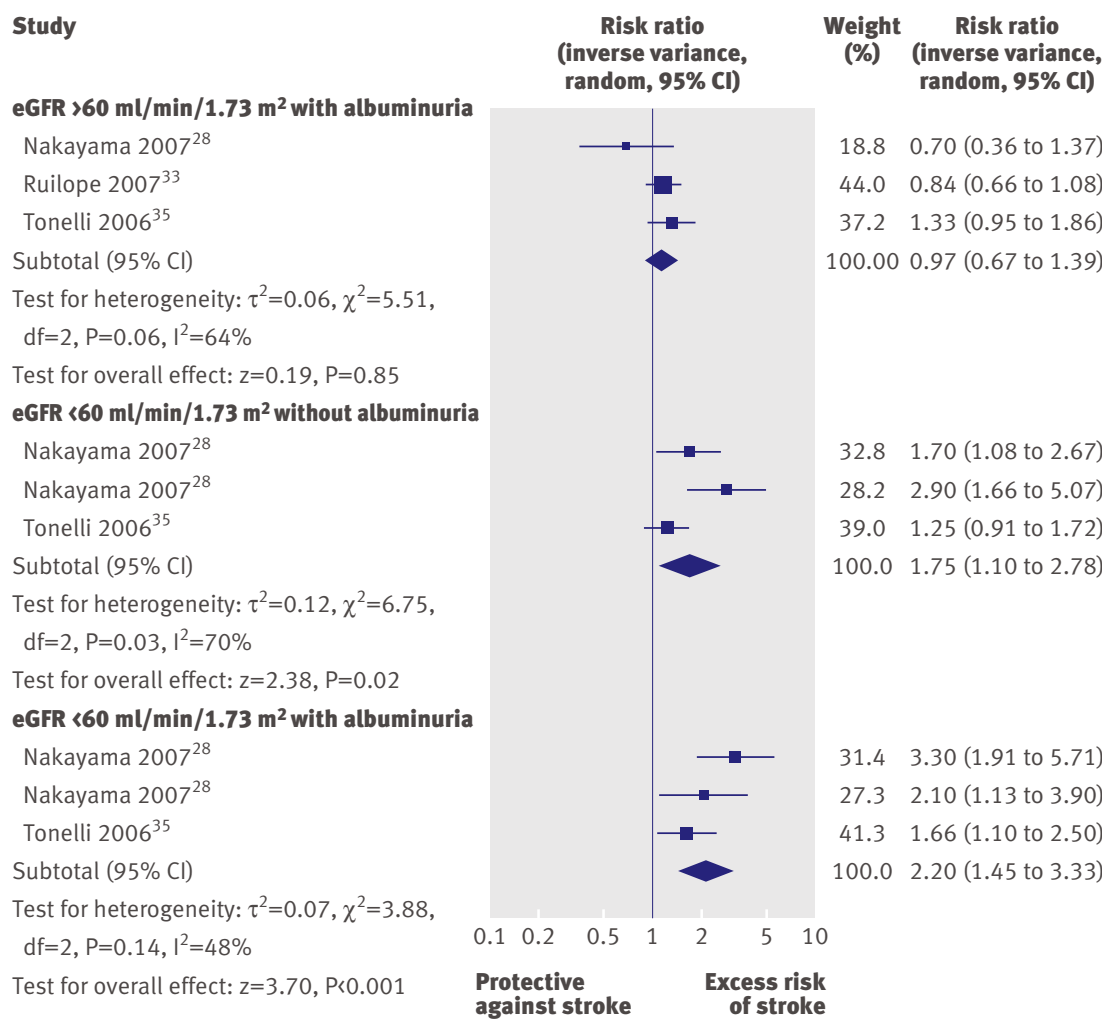

Fig 4 | Interaction between estimated glomerular filtration rate (eGFR) and albuminuria, using groups with eGFR $>60 \mathrm{ml} / \mathrm{min} / 1.73 \mathrm{~m}^{2}$ without albuminuria as reference

\begin{abstract}
$>60 \mathrm{ml} / \mathrm{min} / 1.73 \mathrm{~m}^{2}$. Our result should be interpreted with caution, however, as it was based on just three studies and the rate of albuminuria is low in people without diabetes. A recent meta-analysis showed that compared with people without albuminuria or a low eGFR, those with either condition had a higher risk of cardiovascular death and those with both conditions had the highest risk of cardiovascular death. ${ }^{6}$ Furthermore, meta-analyses have shown that albuminuria was independently associated with a higher risk of stroke even when the included studies had adjusted for eGFR or serum creatinine level. ${ }^{4546}$
\end{abstract}

\section{WHAT IS ALREADY KNOWN ON THIS TOPIC}

Most patients with an estimated glomerular filtration rate (eGFR) $<60 \mathrm{ml} / \mathrm{min} / 1.73 \mathrm{~m}^{2}$ die of cardiovascular causes and not progression to end stage renal disease

A recent meta-analysis showed that an eGFR $<60 \mathrm{ml} / \mathrm{min} / 1.73 \mathrm{~m}^{2}$ was associated with all cause and cardiovascular mortality in the general population

\section{WHAT THIS STUDY ADDS}

People with a baseline eGFR $<60 \mathrm{ml} / \mathrm{min} / 1.73 \mathrm{~m}^{2}$ had an independent risk of future stroke that was $43 \%$ greater than those with a normal baseline eGFR

A dose-response relation between eGFR $<60 \mathrm{ml} / \mathrm{min} / 1.73 \mathrm{~m}^{2}$ and risk of stroke was observed, with risk of stroke being significantly greater for levels $<40 \mathrm{ml} / \mathrm{min} / 1.73 \mathrm{~m}^{2}$ compared with 40-60 ml/min/1.73 $\mathrm{m}^{2}$

Asian patients with an eGFR $<60 \mathrm{ml} / \mathrm{min} / 1.73 \mathrm{~m}^{2}$ were at higher risk of stroke than people of non-Asian ethnicity

\section{Limitations of this meta-analysis}

Limitations of this meta-analysis must be considered. Firstly, meta-analyses may be biased if the literature search fails to identify all relevant studies or the selection criteria for including a study are applied in a subjective manner. To minimise these risks we carried out thorough searches across different databases using explicit criteria for study selection, data abstraction, and data analysis. Secondly, compared with studies of good quality, those of fair quality showed a stronger association between reduced eGFR and stroke. When we restricted analysis to good quality studies, the estimate of association slightly decreased. Thirdly, a large amount of heterogeneity was observed in the results of the various studies. Although subanalyses were done to identify this, heterogeneity persisted in many subgroups, suggesting that other factors might explain this result. Meta-regression by average baseline eGFR and other variables could have been a better way of exploring potential sources of heterogeneity. However, most included articles did not provide average baseline eGFR in each eGFR category, which prevented us from exploring further. In those studies that provided both age and sex adjusted and multivariate unadjusted estimates, the association between reduced eGFR and stroke was slightly, but significantly, attenuated after further adjustment. Such an attenuation in effect size suggests that residual confounding may have remained and that the summary result presented here may be a slight overestimation of the true magnitude of the association between reduced eGFR and risk of stroke. Despite these limitations, the results of this systematic review represent the most precise and accurate estimate of the strength of the relation between reduced eGFR and incident stroke currently available.

\section{Implications}

Our formal meta-analysis found a significant association between eGFR $<60 \mathrm{ml} / \mathrm{min} / 1.73 \mathrm{~m}^{2}$ and increased incident stroke across various populations, after adjustment for established cardiovascular risk factors. None the less, these results possibly underestimated the magnitude of this relation because a reduced eGFR often simultaneously exists with several traditional and novel vascular risk factors. Of major public health interest were our findings that Asian patients with a low eGFR were at higher risk for stroke than their non-Asian counterparts, that below an eGFR level of $60 \mathrm{ml} / \mathrm{min} / 1.73 \mathrm{~m}^{2}$ a dose-response relation with risk of stroke might exist, and that fatal strokes were especially associated with low baseline eGFR.

At this juncture, a low baseline eGFR should be seen simply as a risk marker. Established evidence based strategies already proved to mitigate vascular risk, such as reduction of blood pressure, when promptly and appropriately applied are likely to avert future strokes in people with renal insufficiency. Specific patient subgroups with a low eGFR, such as people of Asian race, may particularly benefit.

We thank Yueh Lee for retrieving the papers 
Contributors: ML and BO conceived the study. ML, S-CC, and BO design the inclusion and exclusion criteria. ML, and K-HC participated in the study search and data collection and extraction. ML did the statistical analysis with guidance from JLS, S-CC, and BO. ML wrote the first draft of the report, and JLS, $\mathrm{H}-\mathrm{WL}$, and $\mathrm{BO}$ did the major revision and made comments. All other authors commented on the draft and approved the final version. ML and BO had full access to all the data and had the final decision to submit for publication. They are guarantors.

Funding: ML was supported by a grant from Chang Gung Memorial Hospital, Taiwan (CMRPG 660311, Taiwan). JLS was supported by the specialised programme on translational research in acute stroke (SPOTRIAS) award (P50 NS044378) from the National Institutes of Health, and BO was supported by University of California, Los AngelesResource Centers for Minority Aging Research under National Institutes of Health/National Institutes on Aging grant No P30-AG021684. The sponsors played no role in the study design, data collection and analysis, or decision to submit the article for publication.

Competing interests: All authors have completed the Unified Competing Interest form at www.icmje.org/coi_disclosure.pdf (available on request from the corresponding author) and declare: no support from any company for the submitted work; no financial relationships with any companies that might have an interest in the submitted work in the previous 3 years; no other relationships or activities that could appear to have influenced the submitted work

Ethical approval: Not required.

Data sharing: No additional data available.

1 Rosamond W, Flegal K, Friday G, Furie K, Go A, Greenlund K, et al. Heart disease and stroke statistics-2007 update: a report from the American Heart Association Statistics Committee and Stroke Statistics Subcommittee. Circulation 2007;115:e69-171.

2 Sarnak MJ, Levey AS, Schoolwerth AC, Coresh J, Culleton B, Hamm LL, et al. Kidney disease as a risk factor for development of cardiovascular disease: a statement from the American Heart Association Councils on Kidney in Cardiovascular Disease, High Blood Pressure Research, Clinical Cardiology, and Epidemiology and Prevention. Circulation 2003;108:2154-69.

3 Schiffrin EL, Lipman ML, Mann JF. Chronic kidney disease: effects on the cardiovascular system. Circulation 2007:116:85-97.

4 Brosius FC, Hostetter TH, Kelepouris E, Mitsnefes MM, Moe SM, Moore MA, et al. Detection of chronic kidney disease in patients with or at increased risk of cardiovascular disease: a science advisory from the American Heart Association Kidney and Cardiovascular Disease Council; the Councils on High Blood Pressure Research, Cardiovascular Disease in the Young, and Epidemiology and Prevention; and the Quality of Care and Outcomes Research Interdisciplinary Working Group: developed in collaboration with the National Kidney Foundation. Circulation 2006:114:1083-7.

5 Di Angelantonio E, Danesh J, Eiriksdottir G, Gudnason V. Renal function and risk of coronary heart disease in general populations: new prospective study and systematic review. PLoS Med 2007;4:e270.

6 Association of estimated glomerular filtration rate and albuminuria with all-cause and cardiovascular mortality in general population cohorts: a collaborative meta-analysis. Lancet 2010;375:2073-81.

7 James MT, Hemmelgarn BR, Tonelli M. Early recognition and prevention of chronic kidney disease. Lancet 2010;375:1296-309.

8 Strippoli GF, Navaneethan SD, Johnson DW, Perkovic V, Pellegrini F, Nicolucci A, et al. Effects of statins in patients with chronic kidney disease: meta-analysis and meta-regression of randomised controlled trials. BMJ 2008:336:645-51.

9 Stroup DF, Berlin JA, Morton SC, Olkin I, Williamson GD, Rennie D, et al. Meta-analysis of observational studies in epidemiology: a proposal for reporting. Meta-analysis Of Observational Studies in Epidemiology (MOOSE) group. JAMA 2000;283:2008-12.

10 Harris RP, Helfand M, Woolf SH, Lohr KN, Mulrow CD, Teutsch SM et al. Current methods of the US Preventive Services Task Force: a review of the process. Am J Prev Med 2001;20:21-35.

11 Loosemore M, Knowles CH, Whyte GP. Amateur boxing and risk of chronic traumatic brain injury: systematic review of observational studies. BMJ 2007;335:809.

12 Smith GL, Lichtman JH, Bracken MB, Shlipak MG, Phillips CO, DiCapua P, et al. Renal impairment and outcomes in heart failure: systematic review and meta-analysis. / Am Coll Cardiol 2006;47:1987-96

13 Higgins JP, Thompson SG, Deeks JJ, Altman DG. Measuring inconsistency in meta-analyses. BMJ 2003:327:557-60.

14 Higgins JP, Thompson SG. Quantifying heterogeneity in a metaanalysis. Stat Med 2002;21:1539-58.

15 Higgins JPT, Green S. Cochrane handbook for systematic reviews of interventions. Cochrane Collaboration, 2008.

16 Ho WK, Hankey GJ, Quinlan DJ, Eikelboom JW. Risk of recurrent venous thromboembolism in patients with common thrombophilia: a systematic review. Arch Intern Med 2006;166:729-36.
17 Critchley JA, Capewell S. Mortality risk reduction associated with smoking cessation in patients with coronary heart disease: a systematic review. JAMA 2003;290:86-97.

18 Bax L, Algra A, Mali WP, Edlinger M, Beutler JJ, van der Graaf Y. Renal function as a risk indicator for cardiovascular events in 3216 patients with manifest arterial disease. Atherosclerosis 2008;200:184-90.

19 Bos MJ, Koudstaal PJ, Hofman A, Breteler MM. Decreased glomerular filtration rate is a risk factor for hemorrhagic but not for ischemic stroke: the Rotterdam Study. Stroke 2007;38:3127-32.

20 Cheng TY, Wen SF, Astor BC, Tao XG, Samet JM, Wen CP. Mortality risks for all causes and cardiovascular diseases and reduced GFR in a middle-aged working population in Taiwan. Am / Kidney Dis 2008;52:1051-60

21 Deo R, Fyr CL, Fried LF, Newman AB, Harris TB, Angleman S, et al. Kidney dysfunction and fatal cardiovascular disease-an association independent of atherosclerotic events: results from the Health, Aging, and Body Composition (Health ABC) study. Am Heart J 2008;155:62-8

22 Ford I, Bezlyak V, Stott DJ, Sattar N, Packard C], Perry I, et al. Reduced glomerular filtration rate and its association with clinical outcome in older patients at risk of vascular events: secondary analysis. PLOS Med 2009;6:e16.

23 Go AS, Fang MC, Udaltsova N, Chang Y, Pomernacki NK, Borowsky L, et al. Impact of proteinuria and glomerular filtration rate on risk of thromboembolism in atrial fibrillation: the anticoagulation and risk factors in atrial fibrillation (ATRIA) study. Circulation 2009;119:1363-9.

24 Irie F, Iso H, Sairenchi T, Fukasawa N, Yamagishi K, Ikehara S, et al. The relationships of proteinuria, serum creatinine, glomerular filtration rate with cardiovascular disease mortality in Japanese general population. Kidney Int 2006;69:1264-71.

25 Kokubo Y, Nakamura S, Okamura T, Yoshimasa Y, Makino H, Watanabe M, et al. Relationship between blood pressure category and incidence of stroke and myocardial infarction in an urban Japanese population with and without chronic kidney disease: the Suita Study. Stroke 2009;40:2674-9.

26 Koren-Morag N, Goldbourt U, Tanne D. Renal dysfunction and risk of ischemic stroke or TIA in patients with cardiovascular disease. Neurology 2006;67:224-8

27 Kurth T, de Jong PE, Cook NR, Buring JE, Ridker PM. Kidney function and risk of cardiovascular disease and mortality in women: a prospective cohort study. BMJ 2009;338:b2392.

28 Nakayama M, Metoki H, Terawaki H, Ohkubo T, Kikuya M, Sato T, et al. Kidney dysfunction as a risk factor for first symptomatic stroke events in a general Japanese population-the Ohasama study. Nephrol Dial Transplant 2007;22:1910-5.

29 Nickolas TL, Khatri M, Boden-Albala B, Kiryluk K, Luo X, Gervasi-Franklin P, et al. The association between kidney disease and cardiovascular risk in a multiethnic cohort: findings from the Northern Manhattan Study (NOMAS). Stroke 2008:39:2876-9.

30 Ninomiya T, Kiyohara Y, Tokuda Y, Doi Y, Arima H, Harada A, et al. Impact of kidney disease and blood pressure on the development of cardiovascular disease: an overview from the Japan Arteriosclerosis Longitudinal Study. Circulation 2008;118:2694-701.

31 Perticone F, Sciacqua A, Maio R, Perticone M, Laino I, Bruni R, et al. Renal function predicts cardiovascular outcomes in southern Italian postmenopausal women. Eur J Cardiovasc Prev Rehabil 2009;16:481-6.

32 Ruilope LM, Salvetti A, Jamerson K, Hansson L, Warnold I, Wedel H, et al. Renal function and intensive lowering of blood pressure in hypertensive participants of the hypertension optimal treatment (HOT) study. J Am Soc Nephrol 2001:12:218-25.

33 Ruilope LM, Zanchetti A, Julius S, McInnes GT, Segura J, Stolt P, et al. Prediction of cardiovascular outcome by estimated glomerular filtration rate and estimated creatinine clearance in the high-risk hypertension population of the VALUE trial. / Hypertens 2007;25:1473-9.

34 Shlipak MG, Simon JA, Grady D, Lin F, Wenger NK, Furberg CD. Rena insufficiency and cardiovascular events in postmenopausal women with coronary heart disease. / Am Coll Cardiol 2001;38:705-11.

35 Tonelli M, Jose P, Curhan G, Sacks F, Braunwald E, Pfeffer M. Proteinuria, impaired kidney function, and adverse outcomes in people with coronary disease: analysis of a previously conducted randomised trial. $B M J$ J 2006;332:1426

36 Weiner DE, Tighiouart H, Amin MG, Stark PC, MacLeod B, Griffith IL, et al. Chronic kidney disease as a risk factor for cardiovascular disease and all-cause mortality: a pooled analysis of communitybased studies. J Am Soc Nephrol 2004:15:1307-15.

37 Yang X, So WY, Ma RC, Ko GT, Kong AP, Ho CS, et al. Thresholds of risk factors for ischemic stroke in type 2 diabetic patients with and without albuminuria: a non-linear approach. Clin Neurol Neurosurg 2008;110:701-9.

38 Perkovic V, Ninomiya T, Arima H, Gallagher M, Jardine M, Cass A, et al. Chronic kidney disease, cardiovascular events, and the effects of perindopril-based blood pressure lowering: data from the PROGRESS study. J Am Soc Nephrol 2007;18:2766-72.

39 Nakamura K, Barzi F, Lam TH, Huxley R, Feigin VL, Ueshima H, et al. Cigarette smoking, systolic blood pressure, and cardiovascular diseases in the Asia-Pacific region. Stroke 2008;39:1694-702 
40 O'Seaghdha CM, Perkovic V, Lam TH, McGinn S, Barzi F, Gu DF, et al. Blood pressure is a major risk factor for renal death: an analysis of 560352 participants from the Asia-Pacific region. Hypertension 2009;54:509-15.

41 Zhang XF, Attia J, D’Este C, Ma XY. The relationship between higher blood pressure and ischaemic, haemorrhagic stroke among Chinese and Caucasians: meta-analysis. Eur J Cardiovasc Prev Rehabil 2006;13:429-37.

42 Hohn AR, Dwyer KM, Dwyer JH. Blood pressure in youth from four ethnic groups: the Pasadena Prevention Project. J Pediatr 1994;125:368-73.

43 Ovbiagele B, Sanossian N, Liebeskind DS, Kim D, Ali LK, Pineda S, et al. Indices of kidney dysfunction and discharge outcomes in hospitalized stroke patients without known renal disease. Cerebrovasc Dis 2009;28:582-8.

44 Ani C, Ovbiagele B. Relation of baseline presence and severity of renal disease to long-term mortality in persons with known stroke. $f$ Neurol Sci 2010;288:123-8.

45 Ninomiya T, Perkovic V, Verdon C, Barzi F, Cass A, Gallagher M, et al. Proteinuria and stroke: a meta-analysis of cohort studies. Am J Kidney Dis 2009;53:417-25.

46 Lee M, Saver JL, Chang KC, Ovbiagele B. Level of albuminuria and risk of stroke: systematic review and meta-analysis. Cerebrovasc Dis 2010 (in press)

Accepted: 25 June 2010 